\title{
FREGE Y LA LÓGICA MODERNA
}

\author{
Carlos Alvarado de Piérola \\ Universidad Nacional Mayor de San Marcos \\ calvaradodep@yahoo.es
}

\section{RESUMEN}

Gottlob Frege constituye un hito en la historia de la lógica. Matemático de profesión, partiendo de la convicción de que las matemáticas estaban deficientemente fundamentadas, desarrolló su obra en la idea de que la aritmética se reduce a la lógica, construyendo en el camino una nueva disciplina: la lógica matemática. En este campo realizó trascendentales aportes que lo colocan junto al fundador de la ciencia que fundara Aristóteles.

\section{PALABRAS CLAVE}

Gottlob Frege/Lógica Moderna/Filosofía de las matemáticas

\begin{abstract}
Gottlob Frege constitutes a milestone in the history of the logic. Mathematician by profession, based on the conviction that mathematics were poorly founded, development work on the idea that the arithmetic is reduced to the logic, building on the road a new discipline: the mathematical logic. In this field made transcendental contributions that it placed next to the founder of the science that founded Aristotle.
\end{abstract}

\section{KEYWORDS}

Gottlob frege/ modern logic/ philosophy of mathematics 


\section{BREVE BIOGRAFÍA}

Friedrich Ludwig Gottlob Frege nació en Wismar, pequeña ciudad portuaria del mar Báltico, en Meklenburgo, Alemania, el 8 de noviembre de 1848. Inició sus estudios universitarios en 1868, en Jena, donde tuvo como profesores a Ernst Abbe y Karl Snell, el primero de los cuales se convertiría en su benefactor. Allí cursó cuatro semestres antes de pasar, en 1871, a la Universidad de Gotinga, en la cual obtuvo el grado de doctor, en 1873, con una tesis «Sobre una representación geométrica de las figuras imaginarias en el plano». Al año siguiente, solicitó y obtuvo un puesto de Privatdozent $^{1}$ en la Universidad de Jena, siendo decano de la Facultad el conocido biólogo darwinista Ernst Haeckel. Allí trabajó hasta 1918, año en que se jubiló. ${ }^{2}$ Se trasladó entonces a Mecklemburgo, donde falleció el 26 de julio de 1925.

\section{LA OBRA DE FREGE}

Su obra se encuadra en el contexto de un gran proyecto: el logicismo. Frege, en controversia con la tendencia psicologista, ${ }^{3}$ fue un defensor de la tesis según la cual las matemáticas son reducibles a la lógica, en el sentido de que las verdades de la matemática son deducibles de las verdades de la lógica. Si calcular es deducir entonces la aritmética se reduce a la lógica. ${ }^{4}$ Es en este contexto que publicó las siguientes obras:

- Begriffsschrift, eine der arithmetischen nachgebildete Formelsprache des reinen Denkens (1879). (Conceptografia: un lenguaje formalizado del pensamiento puro a base del lenguaje aritmético)

- Die Grundlagen der Arithmetik, eine logisch-mathematische Untersuchung über den Begriff der Zahl (1884, 2a. ed. 1934). (Los fundamentos de la aritmética. Investigación lógico-matemática sobre el concepto de número)

- Funktion und Begriff (1891), (Función y concepto). Conferencia del 9 de enero de 1891 en la Jenaische Gesellschaft.

1 Docente sin sueldo.

2 Sin que se le haya concedido jamás una cátedra strictu sensu, según señalan sus biógrafos.

3 Según la tendencia psicologista, la lógica venía a ser parte o rama de la Psicología, y a ella debía sus fundamentos teoréticos.

4 Jesús Mosterín, «Introducción» a Gottlob Frege, Escritos filosóficos. Se sabe, sin embargo, que Frege no fue el primero en formular el proyecto logicista; antes que él, otros, como el matemático alemán Richard Dedekind ya lo habían hecho. 
- «Uber Sinn und Bedeutung», Zeitschrift für Philosophie und philosophische Kritk, N. F. C. (1892), (Sobre significado y referencia).

- «Über Begriffund Gegenstand», Vierteljahrschrift für wissenschaftliche Philosophie, XVI (1892), (Sobre concepto y objeto).

- Grundgesetze der Arithmetik, begriffschriftlich abgeleitet, 2 vols., (18931903). (Leyes básicas de la aritmética ideográficamente deducidas).- la aritmética solo mereció tres críticas, todas ellas hostiles, y permaneció ignorado durante casi veinte años. ${ }^{5}$ Asimismo, vio rechazados sus artículos por las revistas especializadas y los editores desconfiaron de sus proyectos (hasta el extremo de que tuvo que pagar de su propio bolsillo la edición del segundo volumen de sus Leyes básicas de la aritmética).

Cuando, en el primer cuarto del siglo veinte, la lógica matemática comenzó a desarrollarse, pocos eran conscientes de cuánto le debían a Frege por haberlo iniciado. Pero hoy en día ha llegado a ocupar un lugar importante y se ha hecho acreedor al reconocimiento que en vida se le negó. Se considera que su más grande contribución fue en el campo de la lógica y la filosofía de la lógica. Su introducción de los cuantificadores como notación para expresar generalidad provocó una decisiva ruptura entre la lógica moderna y la antigua tradición lógica, a la cual George Boole ${ }^{6}$ y sus seguidores todavía pertenecían. Incluso, se considera a Frege como el primero de los modernos filósofos de las matemáticas y del lenguaje. Dentro de los límites de este artículo, dado su carácter de divulgación, nos ocuparemos de parte de dichas contribuciones.

\section{ETAPAS EN EL PENSAMIENTO DE FREGE}

Según Michael Dummet, la carrera intelectual de Frege puede dividirse en cinco períodos:

- $\quad 1879$, Begriffssschrift.

- Hasta 1884, Die Grundlagen der Arithmetik.

- Hasta 1903, Die Grundgesetze der Arithmetik (II volumen).

- Desde 1903 hasta el año siguiente.

- Desde 1904 hasta su muerte. ${ }^{7}$

5 Anthony Kenny, Introducción a Frege.

6 En su Mathematical Analysis of Logic, George Boole (1815-1864) había desarrollado la idea según la cual las fórmulas algebraicas podían ser usadas para expresar relaciones lógicas, idea que se le ocurrió cuando no había cumplido aún los veinte años de edad. (W. y M. Kneale, El desarrollo de la lógica.)

7 Michael Dummett, Truth and Other Enigmas. 


\section{EL LUGAR DE FREGE EN LA HISTORIA DE LA LÓGICA MATEMÁTICA}

De acuerdo al conocido historiador de la lógica I. M. Bochenski, la historia de la lógica matemática puede dividirse en cuatro períodos.

- Prehistoria: De Leibniz a 1847. En este período surgió la idea de una Lógica matemática, y se formularon numerosos conceptos particulares, sobre todo por Leibniz. No existía aún una escuela de Lógica matemática.

- El periodo de Boole, que va del Analysis ${ }^{8}$ de dicho autor, las Vorlesungen (vol. I, 1895) de Schröder?. Durante este período tiene lugar un desarrollo progresivo de la primera forma de la Lógica matemática, que se diferencia de la posterior, sobre todo, en que sus representantes no hacen objeto de la investigación los métodos de las matemáticas, sino que los aplican sencillamente a la Lógica.

- El periodo de Frege, que va de su Begriffsshrift (1879) a los Principia Mathemática de Whithead y Russell (1910-1913). Durante este periodo, Peirce y Peano, al mismo tiempo que Frege, propusieron una nueva meta en Lógica matemática: la fundamentación de las matemáticas. En él se desarrollan una serie de importantes conceptos y métodos lógicos.

- El periodo contemporáneo, que parte de los Principia y se encuentra todavía sin concluir.(Bochenski, 1966, pp. 284-286).

Como se ve, Gottlob Frege inaugura una etapa y se constituye en un hito histórico importante en la historia de la lógica. Su Annus Mirabilis es el año 1879, fecha de la publicación de su obra fundamental, Conceptografía. Esta es una opinión en torno a la cual existe un significativo consenso, considerándose a Frege como el protagonista de una revolución similar a la copernicana en este campo. Si consideramos a Aristóteles como el creador de la lógica, creación que permaneció vigente durante más de dos mil años, la obra de Frege es solo comparable con la del Estagirita. Contrariamente a lo que había señalado Kant, quien pensaba que la lógica había llegado a su máximo desarrollo y le negaba la posibilidad de un novedoso futuro (el famoso «error histórico» de Kant), con el autor de la Conceptografía, la lógica renació con una fuerza sorprendente y arrolladora, iniciando un desarrollo que continúa hasta nuestros días. Anthony Kenny dice al respecto: «Si Aristóteles fue el fundador de la lógica, Frege la volvió a fundar; y en el periodo que va desde su tiempo hasta el día de hoy, la lógica ha avanzado más rápida y profundamente

8 Mathematical Analysis of Logic . Publicada por Boole en 1847.

9 El ya mencionado libro titulado Vorlesungen über die Algebra der Logik (Lecciones sobre el Álgebra de la Lógica), publicada por Ernst Schröder en tres volúmenes. Gracias a él, se generalizó el término lógica matemática. 
de lo que hizo durante todas las centurias que separaron a Frege de Aristóteles». (Kenny, 1997, p. 269).

\section{EL PROYECTO LOGICISTA}

Como ya lo hemos mencionado, nuestro personaje fue un defensor de la tesis según la cual las matemáticas, particularmente la aritmética, son reducibles a la lógica. ${ }^{10}$ Mientras que Boole había tratado de presentar a la lógica como una parte de la matemática, Frege consideraba que la aritmética se identifica con la lógica. ${ }^{11}$ Como señala Mosterín (2000): Específicamente, el objetivo era definir las nociones aritméticas de la aritmética a partir de principios lógicos, deduciendo los teoremas de la aritmética a partir de principios lógicos. Pero para poder llevar adelante este proyecto, necesitaba de una herramienta de la cual no disponía: un lenguaje adecuado. Como tarea previa, se dedicó afanosamente a la realización de este objetivo. Así es como apareció la Conceptografía, que contenía importante trabajo preparatorio para la pretendida reducción de la aritmética a la lógica. En esta obra, Frege establece un nuevo cálculo en cuya exposición introduce también una serie de profundas observaciones sobre la naturaleza de la lógica, de la prueba y del lenguaje. Lo presenta en un simbolismo inventado para tal fin. ${ }^{12}$ Partía de la idea según la cual el lenguaje ordinario no era lo suficientemente preciso para

10 Como se sabe, la defensa de Frege del logicismo era de alcance limitado, aplicándola sólo a la aritmética, puesto que permaneció kantiano respecto a la geometría.

11 A primera vista, los dos programas parecen contrapuestos, y los filósofos poco propicios a uno y otro han apuntado burlonamente esta aparente contradicción. Cuando Boole programaba el análisis matemático de la lógica, pensaba únicamente en la presentación de la lógica como un cálculo bajo ciertos aspectos semejante al álgebra numérico. Como Leibniz, consideraba que el cometido específico de la ciencia matemática era la construcción de cálculos y confiaba en la posibilidad de elaborar interesantes cálculos susceptibles de ser interpretados sin referencia al número o la cantidad. Frege no deseaba, por su parte, negar tal posibilidad. Por el contrario, fue mucho más lejos que cualquiera de sus predecesores en su exigencia de rigor formal en el estudio de la lógica, y el cálculo o sistema deductivo por él elaborado es la mayor hazaña consumada por autor alguno en la historia de nuestra disciplina. Pero trató también de hacer ver que los números podían ser definidos sin referencia a otras nociones que las envueltas en la interpretación de su cálculo como un sistema de lógica. De estar Frege en lo cierto, la lógica incluiría la aritmética y con ella todas aquellas ramas de la matemática que quepa reducir a la aritmética e el sentido de poderse demostrar que no envuelven tampoco otras nociones que las que ya se encuentran en esta última o la lógica.” (W. y M. Kneale, Op. Cit., p. 402.)

12 Dadas las limitaciones a las que nos enfrentamos, no es posible exponer aquí el simbolismo de Frege, que, dicho sea de paso, tiene ahora solo un valor histórico pues no se utiliza. 
sus propósitos. Se necesitaba un nuevo lenguaje, que superara estas limitaciones, por eso inventó la escritura conceptual. Los elementos de los enunciados que son esenciales para la inferencia constituyen, en la terminología de Frege, el «contenido conceptual».(Kenny, p. 269)

La construcción de este simbolismo estaba originalmente motivada por su deseo de establecer con rigor la verdadera naturaleza de la aritmética. Considerando que las leyes de la lógica operan en cualquier esfera del conocimiento, ¿descansan las pruebas aritméticas puramente en estas leyes lógicas, o necesitan el apoyo de hechos empíricos? Para responder a esta cuestión, habría que ver «hasta dónde se podría llegar en aritmética sólo con deducciones lógicas, apoyándose únicamente en las leyes del pensamiento». En 1884 salió a la luz su obra Die Grundlagen der Arithmetik, eine logisch-mathematische Untersuchung über den Begriff der Zahl (Los fundamentos de la aritmética. Investigación lógico-matemática sobre el concepto de número), a la cual Michael Dummmet considera su obra maestra. En dicha obra escribió:

Espero haber hecho verosímil en esta obra la idea de que las leyes aritméticas son juicios analíticos y que, por consiguiente, son a priori. La aritmética, por tanto, sería solamente una lógica más extensamente desarrollada, y cada enunciado aritmético sería una ley lógica, aunque una ley derivada. Las aplicaciones de la aritmética en la explicación de la naturaleza serían elaboraciones lógicas de hechos observados; $(\ldots) .^{13}$

Consecuente con estas convicciones, en su Grundgesetze der Arithmetik (Leyes básicas de la aritmética) intenta derivar las leyes de la aritmética a partir de la lógica. Frege la consideraba la obra mayor de su vida; pero, entonces, sucede un acontecimiento totalmente inesperado: en 1902, con las pruebas corregidas del segundo volumen ya en la imprenta, recibió una carta de Bertrand Russell en la que le advertía acerca de una grave inconsistencia en su sistema lógico, de la Teoría de los conjuntos formulada junto con Cantor y Dedekind, conocida más adelante como la paradoja de Russell. Frege quedó profundamente impresionado; sin embargo, en un intento por salvar su proyecto, introdujo a toda prisa una modificación en uno de sus axiomas, el V, de la que dejó constancia en un apéndice de la obra. ${ }^{14}$ Este golpe a

13 Gottlob Frege, «Los fundamentos de la aritmética», en Escritos filosóficos.

14 Dicho axioma era el siguiente: " $(\dot{\varepsilon} f(\varepsilon)=\dot{\alpha} g(\alpha))=((x) f(x)=g(x))$

«Esta proposición nos dice que si los cursos de valores de dos funciones son idénticos, entonces el valor de una función para un elemento dado es siempre el mismo que el valor de la otra función: podemos dar el paso desde una clase a la función correspondiente. Y a la inversa, también nos dice que si el valor de una función para un argumento dado es siempre el mismo que el valor de la otra función par el mismo 
la estructura de su obra prácticamente puso fin a su actividad académica y arruinaría los sueños logicistas de Frege. Desilusionado, no le quedó más remedio que escribir en un apéndice de Grundgesetze lo siguiente: "Nada más triste puede suceder a un escritor científico que ver cómo, después de haber terminado su trabajo, uno de los fundamentos de su construcción se tambalea". (Mosterín, p. 16).

Al final, Frege dejó de lado el logicismo, en su lugar empezó a considerar la posibilidad de que la geometría podría fundamentar a la aritmética. (p. 16).

\section{LOS APORTES DE FREGE A LA LÓGICA MODERNA.}

Frege fue el primero en introducir en lógica la idea de proposición, ${ }^{15}$ en formular con precisión la diferencia entre variable y constante, lo mismo que el concepto de función lógica, la idea de una función pluriargumental, y el concepto de cuantificador; a él se debe una concepción mucho más exacta de la teoría aristotélica de sistema axiomático, al igual que una clara distinción entre ley y regla, lenguaje y metalenguaje; él es el autor de la teoría de la descripción, y si bien no el inventor del concepto de valor, sí el primero en elaborarlo sistemáticamente. ${ }^{16}$

Como ya lo hemos señalado, Frege fue también un importante filósofo del lenguaje. La distinción entre sentido y referencia y entre concepto y objeto se deben a él.

\section{VARIABLE Y CONSTANTE}

Ya Aristóteles distinguía entre expresiones constantes y expresiones variables 0 , más brevemente, constantes y variables:

argumento, entonces los cursos de valores son idénticos: podemos dar el paso desde una función a la clase correspondiente. Frege tomó la paradoja de Russell como evidencia de que no podemos transformar una identidad de cursos de valores en una identidad general. Sin embargo, pensó que la paradoja no destruye la posibilidad de transformar una identidad general en una identidad de cursos de ${ }_{225}$ cursos de valores. De acuerdo con ello modificó el axioma $\mathrm{V}$ de manera que permitiera la transición de una identidad entre clases, pero que no permitiera ya la correspondiente transición entre clases y funciones. Este debilitamiento bloquearía, a su juicio, la formulación de la paradoja russelliana y permitiría sin embargo que la aritmética fuera derivada de la lógica como él proponía.» (Anthony Kenny, Op. Cit., pp. 225-226.

15 Ian Łukasiewicz, La silogistica de Aristóteles. (Aserción: proposición.)

16 I. M. Bochenski, Op. Cit. 
Modo

Si $\boldsymbol{A}$ conviene a todo $\boldsymbol{B}$

Y $\boldsymbol{B}$ conviene a todo $\boldsymbol{C}$,

Entonces $\boldsymbol{A}$ conviene a todo $\boldsymbol{C}$.
Sustitución

Si animal conviene a todo hombre y hombre conviene a todo ser racional, Entonces animal conviene a todo ser racional.

Las primeras tienen un sentido determinado, mientras las otras sirven únicamente para indicar lugares vacíos en los que pueden colocarse constantes. Por ejemplo, en «x lee», «X» es una variable y «lee» es una constante. Frege llamará función a la expresión molecular en la que aparece una variable.

\section{FUNCIÓN LÓGICA Y VALOR DE VERDAD}

En el sistema de Frege la distinción tradicional entre sujeto y predicado queda sustituida por la distinción entre función y argumento, tomada de la terminología de las matemáticas. Veamos cómo lo hizo.

De una expresión algebraica como $\left\langle x^{2}+1 »\right.$ se puede decir que representa una función de $x$ : el valor del número representado por la expresión entera « $\left\langle x^{2}+1 »\right.$ variará de acuerdo con el numeral que escribamos en lugar de la variable $x$. El valor total de la expresión está en función del valor que adquiera $x .{ }^{17}$

Así, el valor de la expresión-función « $x^{2}+1 »$ para el argumento 1 es $2\left(1^{2}\right.$ $+1=1+1=2)$. Para el argumento 2 es $5\left(2^{2}+1=4+1=54\right)$. Para el argumento 3 es $10\left(3^{2}+1=9+1=10\right)$, y así sucesivamente. . $^{18}$

Hay, también, funciones de 2 o más argumentos:

$$
\begin{gathered}
\left\langle x^{2}+\mathrm{y} »\right. \\
\left\langle 2 \mathrm{y} x^{2}+\mathrm{z} »\right.
\end{gathered}
$$

17 "Así como se alude indeterminadamente a un número por medio de una letra, para expresar generalización, asimismo se siente la necesidad de aludir indeterminadamente a una función por medio de letras. Para ello, se suele hacer uso generalmente de las letras $f$ y $F$, de tal manera que, en 'f(x)' y ' $F(x), x$ representa el argumento." ("Función y concepto", en Gottlob Frege, Estudios sobre semántica, p. 28).

Argumento $=$ variable. "(...) para distinguir las relaciones funcionales de dos miembros de las de tres, hablaremos en el primer caso de funciones de una variable o de un argumento; y en el segundo, de funciones de dos variables o de dos argumentos; (...)." (Alfred TARSKI, Introducción a la lógica, p. 120).

18 Argumento 1: la variable $x$ a la cual se le ha otorgado el valor numérico 1. Argumento 2: la variable $x$ a la cual se le ha otorgado el valor numérico 2 . Argumento 3: la variable $x$ a la cual se le ha otorgado el valor numérico 3. 
En cada caso la función tendrá un valor especificado sólo si se rellenan todos los espacios destinados a argumentos, y normalmente el valor será diferente para diferentes argumentos.

Frege hizo dos innovaciones:

1) Extendió la noción de función a ecuaciones liberándola de su restricción a expresiones de números (es decir, la extendió a expresiones como « $x^{2}$ $=1 »$, no reduciéndola a expresiones como « $x^{2}+1 » \mathrm{y}$

2) Igualmente, la extendió a expresiones del lenguaje ordinario, sin restringirla a expresiones puestas en notación matemática.

De esta manera, a expresiones como «la capital de $x »$ las llamó expresiones de una función, que toma como valor Londres cuando Gran Bretaña es el valor del argumento $x$. También de una oración tal como «César conquistó la Galia» consideró que significaba el resultado o bien de completar la función « $x$ conquisto la Galia» con el argumento "César», o bien de completar la función «César conquisto $x »$ con el argumento «Galia», o bien el de completar la función «X conquisto $y$ » con los argumentos «César» y «Galia» en los lugares apropiados.

Del mismo modo, para Frege, «César conquistó la Galia» representa lo Verdadero, y «Pompeyo conquistó la Galia», lo Falso. Los valores de la función «x conquisto la Galia» para distintos argumentos serán siempre un «valor de verdad» (verdadero o falso). ${ }^{19}$

Los enunciados afirmativos pueden concebirse lo mismo que las ecuaciones o las expresiones analíticas, descompuestas en dos partes, una de las cuales está completa en sí misma (saturada), mientras que la otra precisa de complemento (no saturada).

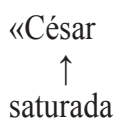

ARGUMENTO FUNCIÓN

19 Por eso, decimos que hay valores de verdad verdaderos y valores de verdad falsos. 
Expresión de una función: «x conquisto la Galia».

Si tomamos como argumento suyo «conquisto la Galia» obtendremos, como valor de la función, César.

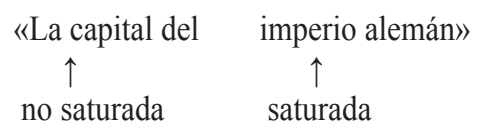

\section{FUNCIÓN $\quad$ ARGUMENTO}

Expresión de una función: «la capital de $x »$.

Si tomamos como argumento suyo el imperio alemán obtendremos, como valor de la función, Berlín.

En ambos casos, la segunda parte (función) lleva consigo un lugar vacío, deja de estarlo cuando se llena con un nombre propio; entonces aparecerá un sentido completo. La función es comprendida relacionalmente, con lugares vacíos que deberán ser llenados por los «argumentos» (números en la aritmética, objetos de cualquier tipo en la lógica).

¿Cuál es el valor de las funciones significadas, por ejemplo, « $x=1 » \mathrm{y} « x$ conquistó la Galia»? La respuesta de Frege es:

Tomemos la expresión «“ $x^{2}=1 »$. Si sustituimos $x$ sucesivamente por -1 , 0,1 y 2 tendremos:

$$
\begin{gathered}
(-1)^{2}=1 \\
0^{2}=1 \\
1^{2}=1 \\
2^{2}=1
\end{gathered}
$$

De estas ecuaciones, la primera y la tercera son verdaderas; las demás, falsas.

Frege llamó a la verdad o falsedad de una ecuación su «valor de verdad» y sugirió que este valor de verdad habría de ser considerado como el valor de la función para los distintos argumentos: 
Así, pues, digo: «el valor de nuestra función es un valor veritativo» y distingo el valor veritativo de lo verdadero y el de lo falso. Para abreviar, a uno lo llamo lo verdadero, y al otro lo falso. Según esto, $« 2^{2}=4 »$, por ejemplo se refiere a lo verdadero, al igual que $\left\langle 2^{2}\right\rangle$, por ejemplo se refiere a 4. $\mathrm{Y} « 2^{2}=1 »$ se refiere a lo falso. (Bochenski, p. 341).

Aunque la idea de valores de verdad existe ya en la escuela megárica, su expresión y primera descripción se debe a Frege. ${ }^{20}$

\section{LOS CUANTIFICADORES}

Frege Introdujo una nueva sintaxis, con la inclusión de los llamados cuantificadores («para todo» o «para algún caso de»).

Un cuantificador se utiliza para indicar cuántos elementos de un conjunto dado cumplen con cierta propiedad. También se dice que un cuantificador es una expresión que afirma que una condición se cumple para un cierto número de individuos. Esta es la más significativa de las invenciones de Frege. Su introducción de los cuantificadores como notación para expresar generalidad provocó una decisiva ruptura entre la lógica moderna y la antigua tradición lógica, a la cual George Boole y sus seguidores todavía pertenecían. Estos son palabras como «todos/as», «algunos/as», «muchos/as», y «la mayoría de», que nos permiten construir enunciados sobre grupos de objetos. Por ejemplo: Algunos hombres son calvos. Para Aristóteles eran sujetos de predicación en un enunciado que puede conducir a absurdos, como en este diálogo de Alicia en el país de las Maravillas:

- A nadie veo en el camino - dijo Alicia.

- Ojalá tuviera unos ojos así - se lamentó el rey. -Ser capaz de ver a nadie. Y además a esa distancia. Vaya... Bastante hago con ver gente real.

Frege evita este problema tratando los cuantificadores como entidades lógicamente independientes. Usando «todos/as» y «hay al menos un/a» logra traducir «a nadie veo en el camino» como:

- Para todas las personas, no puedo verlas en el camino.

- No hay al menos una persona tal que pueda verla en el camino.

Nos dice que la palabra nadie no tiene por qué referirse a un objeto. (Kenny, p. 269).

20 I. M. (Bochenski, p. 341). 
Existencial: el segundo que se cumple para al menos uno de los individuos

Esta maquinaria puede adaptarse fácilmente para formalizar oraciones con cuantificadores del lenguaje natural. Tómese por caso la afirmación «todos son amigables». Esta oración puede traducirse así:

Para todo $x, x$ es amigable.

Y una oración como «alguien está mintiendo» puede traducirse:

Para al menos un $x, x$ está mintiendo.

También es frecuente traducir esta última oración así:

Existe al menos un $x$, tal que $x$ está mintiendo.

A continuación se formalizan ambas oraciones, introduciendo a la vez la notación especial para los cuantificadores: ${ }^{21}$

Para todo $x, x$ es amigable:

$(\forall \mathrm{x}) \mathrm{Ax}$

Existe al menos un $x$, tal que $x$ está mintiendo:

$(\exists \mathrm{x}) \mathrm{Mx}$

Entre los historiadores de la lógica, existe consenso en señalar que la introducción de los cuantificadores por parte de Frege constituye la novedad más importante. Así, W. y M. Kneale afirman: «(...) no sería exagerado calificar al empleo de cuantificadores para ligar variables como una de las grandes conquistas intelectuales del siglo diecinueve». ${ }^{1}$ Kenny comparte este criterio: «La mayor contribución de Frege a la lógica fue su invención de la teoría de la cuantificación». ${ }^{2}$ A su vez, Michael Dummett, señala: «Su introducción de la notación con cuantificadores y variables para expresar la generalidad provocó el claro rompimiento entre la lógica moderna y la antigua tradición lógica a la cual todavía pertenecen George Boole y sus seguidores». ${ }^{3}$

\section{SISTEMA AXIOMÁTICO.}

Frege inició el verdadero comienzo de la moderna lógica matemática al haber creado el primer «cálculo lógico» en el sentido estricto del término en matemáticas. En la Lógica matemática, el cálculo proposicional de Frege fue la primera axiomatización del cálculo proposicional. Hace uso de sólo dos operadores lógicos: implicación y negación.

21 La notación utilizada es la moderna, pues la original, la de Frege, era otra. 
Su sistema está constituida por nueve axiomas y una regla de inferencia: modus ponens. Frege presenta por primera vez en su Begriffsschrift un cálculo deductivo en su sentido actual (es decir, un sistema formal) para lo lógica de primer y segundo orden.

Predicado de primer orden: sus argumentos son objetos.

Predicados de segundo orden: sus argumentos son predicados de segundo orden.

Esto no sería superado sino hasta 50 años más tarde (con la publicación del libro de Hilbert y Ackermann Grundzuge der theorischen Logik - 1928). ${ }^{22}$

\section{LA NEGACIÓN}

Frege introdujo el signo de negación. Kenny nos recuerda lo que expresa Frege al respecto en su Conceptografia $(\mathrm{C}, \S 7)$ : «expresa la circunstancia de que el contenido [del enunciado] no tiene lugar». Pero debe tomarse en cuenta que la negación no se aplica al predicado gramatical de la oración, sino a la oración en su totalidad. Así, la negación de «Sócrates es mortal» sería, utilizando para la negación el símbolo que conocemos, « (Sócrates es mortal)». Esto se explica por el rechazo de Frege a la distinción gramatical de sujeto y predicado. (Kenny, p. 38).

\section{INFLUENCIAS EN OTROS FILÓSOFOS.}

El trabajo de Frege influyó en los Principa Mathematica de Russell y Whitehead y en Giuseppe Peano, Ludwig Wittgenstein Edmund Husserl.

Más tarde Kurt Gödel en su Teorema de incompletitud demostró que el programa logicista de Frege-Russell era incompleto. Esto es, que todo sistema formal contendrá al menos un enunciado verdadero indemostrable desde el sistema, el así llamado «enunciado de Gödel.»

Quisiéramos concluir esta rápida revista de la obra del pensador de Jena con estas palabras de Kenny:

Así como Colón fracasó en su proyecto de descubrir un camino a la India por el lado oeste, y sin saberlo enriqueció a Europa con el conocimiento

22 Cf. Jesús Mosterín, Op. Cit. 
de todo un nuevo continente, del mismo modo Frege fracasó en su tarea de derivar la aritmética de la lógica, pero realizó descubrimientos en lógica y avances en filosofía que cambiaron de manera permanente el mapa entero de ambas disciplinas. (Kenny, p. 267).

\section{REFERENCIAS BIBLIOGRÁFICAS}

\section{Obras de Frege}

Frege, G. (1972). Conceptografía. Los fundamentos de la aritmética. Otros estudios filosóficos. México: D. F., UNAM.

(1985). Estudios sobre semántica. Madrid: Orbis.

(1996). Escritos filosóficos. Barcelona: Crítica.

Frege-H. (1987). Correspondance, Ed. T. E. R. (para el texto francés) y Felix Meiner Verlag, Hamburg, 1980 (para el texto alemán).

\section{Obras sobre Frege}

Bochenski (1966). Historia de la lógica formal. Madrid: Gredos.

Chávez, A. (2001). Frege: El cálculo lógico. Lima: Ed. del autor.

Dummett, M. (1978). Truth and other enigmas. London: Duckworth.

Kenny, A. (1997). Introducción a Frege. Madrid: Cátedra.

Kneale, W. y M. (1980). El desarrollo de la lógica. Madrid: Tecnos.

Mosterín, J. (2000). Los lógicos. Madrid: Espasa Calpe.

Tarski, A. (1951). Introducción a la lógica. Buenos Aires: Espasa-Calpe. 